\title{
THE STRUCTURE OF THE INTERSTELLAR MEDIUM
}

\author{
JOEL N. BREGMAN and GREGORY A. ASHE \\ Astronomy Department, University of Michigan \\ Ann Arbor, MI 48109-1090, USA
}

\begin{abstract}
We have compared theoretical HI models to a large area $\left(18^{\circ} \times 12^{\circ}\right)$ HI channel map centered at $1=$ $205^{\circ}, \mathrm{b}=0^{\circ}$. One set of models were calculated in which uncorrelated clouds of HI populate space. Of the cloud models considered, which include "Spitzer"-type clouds and the McKee-Ostriker formulation, none were able to reproduce the data successfully. In another class of models, holes and holes with shells permeate an otherwise continuous HI medium. Although not entirely successful, these models contain characteristics that are similar to the data. In order to reproduce the amplitude of the HI variations on angular scales of $>1^{\circ}$, the volume occupied by ionized gas must be less than $30 \%$, in sharp conflict with the models that suggest that the volume of the ISM is dominated by hot gas.
\end{abstract}

\section{INTRODUCTION}

The structure of the interstellar medium in the disk of the galaxy has a profound effect on the evolution of energetic events in the disk, such as supernovae. Supernovae produce hot gas and cosmic rays which, depending upon the structure of the ISM, can escape into the halo. The evolution of supernovae and other energetically important phenomena, such as the closely related and very powerful stellar winds (van Buren 1986), is determined by the properties of the interstellar gas that occupies most of the volume. At this time, we lack a consensus as to the volume occupied by the most common component and its geometry.

\subsection{Theoretical Models}

In theoretical models of the ISM that were developed in the mid-1970s and early 1980s, the principal energetic component was randomly placed supernova remnants (Cox and Smith 1974; McKee and Ostriker 1977; Cox 1981; Cowie, McKee, and Ostriker 1981). These models applied the results of spherically symmetric supernova remnant calculations to the disk in a statistical manner in order to determine the filling factor of the hot supernova remnants. Given the estimates for the supernova rate and the energy deposition per supernova, these efforts suggested that most of the volume was occupied by a hot, dilute gas that was responsible for the soft X-ray emission seen in the Galaxy. In this picture, supernovae shocks propagate mainly through the dilute hot medium, and this hot gas escapes freely into the halo.

A change in this theoretical perspective began when it was argued that supernovae occur primarily in associations of young stars, which give rise to large reheated supernova remnants, known as superbubbles (Weaver et al. 1977; Bruhweiler et al. 1980; Tomisaka and Ikeuchi 1986; McCray and Kafatos 1987; Tenorio-Tagle, Bodenheimer, and Rozyczka 1987; Mac Low and McCray 1988; Tenorio-Tagle and Bodenheimer 1988; Mac Low, McCray, and Norman 1989). Typically, 30 
supernovae lead to a single superbubble, which grows in size to several hundred parsecs (diameter) and eventually breaks out of the disk. If most of the supernovae participate in the growth of superbubbles, then the fractional volume occupied by the hot medium is likely to be less than $50 \%$, possibly in the $10-20 \%$ range (Norman and Ikeuchi 1989).

\subsection{Observational Constraints}

From observations, it was difficult to estimate the filling factor of the various phases of the hot medium. For external galaxies, $\mathrm{X}$-ray observations of disk galaxies failed to detect a diffuse emission component from a hot dilute gas (McCammon and Sanders 1984), although this may simply indicate that the gas is cooler than $6 \times 10^{5} \mathrm{~K}$. A search for HI holes in the nearby galaxies M31 and M33 (Brinks and Shane 1984; Brinks and Bajaja 1986; Deul 1988) was more successful, demonstrating that hundreds of large bubbles could exist in a single galaxy. These surveys, which were sensitive to holes larger than about $100 \mathrm{pc}$ (about the size of a small superbubble, but larger than a single supernova remnant), showed that about $5 \%$ of the surface area of a galaxy was covered by such objects.

In the Milky Way galaxy, several prominent ISM components either have significant mass or volume fractions. For warm ionized gas $\left(10^{3}-10^{4} \mathrm{~K}\right)$, a comparison between the $\mathrm{H} \alpha$ emission measure and the pulsar dispersion measure suggests a volume filling factor of about $20 \%$ (e.g., Cox and Reynolds 1987; Reynolds 1989$)$. For the hot material $\left(10^{6} \mathrm{~K}\right)$, there is an anticorrelation between the soft X-ray emission and the HI. This effect, which can be explained as the displacement of one phase by another, would not have been noticed if either medium occupied an insignificant volume of the ISM. This suggests that both the hot and cold components occupy $>10 \%$ of the volume, although it is difficult to obtain more precise constraints because the phases are not uniform and we detect mainly local hot gas (Cox and Reynolds 1987). The HI bubbles seen by Heiles (1984) is analogous to the HI holes seen in external galaxies and supports the concept of hot gas displacing the $\mathrm{HI}$.

The pervasiveness of the $21 \mathrm{~cm} \mathrm{HI} \mathrm{emission} \mathrm{line} \mathrm{has} \mathrm{prompted} \mathrm{most} \mathrm{observers} \mathrm{to} \mathrm{conclude}$ that this component must occupy a significant amount of the volume (e.g., Kulkarni and Heiles 1988, Dickey and Lockman 1990, and references therein). However, most of these arguments have been qualitative in nature. We have been developing a model that will permit a more quantitative analysis of the volume occupied by the HI and hotter components. This model also permits us to discuss the geometric structure of the HI.

\section{THE MODEL}

Our approach is to calculate HI maps of a part of the disk of the Galaxy that can be compared directly to observations (HI channel maps). A phenomenological model interstellar medium can be constructed by specifying different "forms" that may be present in the HI layer. Possible forms of HI are clouds (with or without a dense core), a uniform medium (with a vertical scale height and radial scale length), holes in the uniform medium, and expanding shells. The properties of each member of each form within the Galaxy are specified (location, random velocity, size, density, etc.) and then the emission image as viewed from the Sun can be calculated and compared to the observed surface brightness distribution. We regard this as a phenomenological model because the gas properties are assumed (or taken from observations) rather than calculated from first principles using detailed hydrodynamics.

In constructing such a model, we require that the mean observed density distribution be reproduced in latitude, longitude, and velocity. In doing so, we adopt the mean HI vertical distribution given by Dickey and Lockman (1990) in which there are two Gaussian distributions 
(FWHM of $h_{1}=212 \mathrm{pc}$ and $h_{2}=530 \mathrm{pc}$ ) and an exponential distribution (scale height $h_{3}=403 \mathrm{pc}$ ). In addition, the flaring of the $\mathrm{HI}$ disk is included by having the scale heights be proportional to the distance:

$$
\begin{array}{ll}
h_{i}(R)=h_{i}\left(R_{\odot}\right) & \text { for } R<R_{\odot} \\
h_{i}(R)=\frac{R}{R_{\odot}} h_{i}\left(R_{\odot}\right) & \text { for } R \geq R_{\odot}
\end{array}
$$

This leads to a final mean distribution for the HI of

$$
n(z)=\left(0.40 e^{-\frac{z^{2}}{2 h_{1}^{2}}}+0.11 e^{-\frac{z^{2}}{2 h_{2}^{2}}}+0.06 e^{-\frac{z^{2}}{h_{3}}}\right) e^{\frac{-\left(R-R_{0}\right)}{h_{R}}}
$$

which implies a total column perpendicular to the disk of $6.2 \times 10^{20} \mathrm{~cm}^{-2}$. This gas is assumed to be in circular rotation about the Galaxy with a velocity of $220 \mathrm{~km} \mathrm{~s}^{-1}$ (flat rotation curve). The warp of the HI layer is not taken into account because the longitude region that will be the point of comparison is nearly along the line of nodes.

We have produced models in the longitude and latitude range $196-214^{\circ}$ and $\pm 6^{\circ}$, which is the region where Lockman and Ganzel (1983) have obtained spectra with 20' resolution. Within this volume, we distribute the interstellar medium in particular forms and then calculate the appearance of the HI emission, producing a three dimensional data cube, with ordinates of longitude, latitude, and velocity. An example of this modeling is an interstellar medium composed of an ensemble of uncorrelated $\mathrm{HI}$ clouds. These clouds are defined by their size, density, internal velocity dispersion, and random velocity. When determining whether a cloud exists at a particular location, a random number generator is first used to select a location. Then, the probability that a cloud will be created is proportional to the mean density $n(R, z)$ given above. Clouds are added to the volume until the total emissivity, integrated over longitude, latitude, and velocity, matches the observed quantity.

From this ensemble of clouds, in order to convert the column density into a brightness temperature $\left(T_{B}\right)$, opacity corrections are introduced. Largely for convenience, we have assumed that the $\mathrm{HI}$ is at a constant temperature of $\mathrm{T}_{\text {spin }}=100 \mathrm{~K}$. The simple radiative transfer equations become

$$
\begin{aligned}
T_{B}(v) & =T_{s p i n}\left(1-e^{-\tau(v)}\right) \Delta v \\
\tau(v) & =\frac{N_{H}(v)}{1.83 \times 10^{18} T_{s p i n}}
\end{aligned}
$$

where $\Delta v$ is the channel width and $v$ is the central velocity of the channel. In practice, we use $\Delta v=$ $2 \mathrm{~km} \mathrm{~s}^{-1}$, and this calculation is carried out for angular bins that are 5' on a side. The image at a particular velocity, $T_{B}(1, b, v)$, is convolved with a Gaussian to a resolution of 20 , which is the resolution of the data set of Lockman and Ganzel.

The resulting models or data cubes are meant to be representative of an ISM with a particular form (e.g., clouds) rather than a detailed reproduction of the observations. Consequently, it is necessary to characterize the models and the data in statistical terms. We have calculated surface area as a function of brightness temperature as well as structure functions and Fourier power spectra of strips across the channel maps. These statistics place on a quantitative footing the information evident to the eye in Figures 1, 2. 

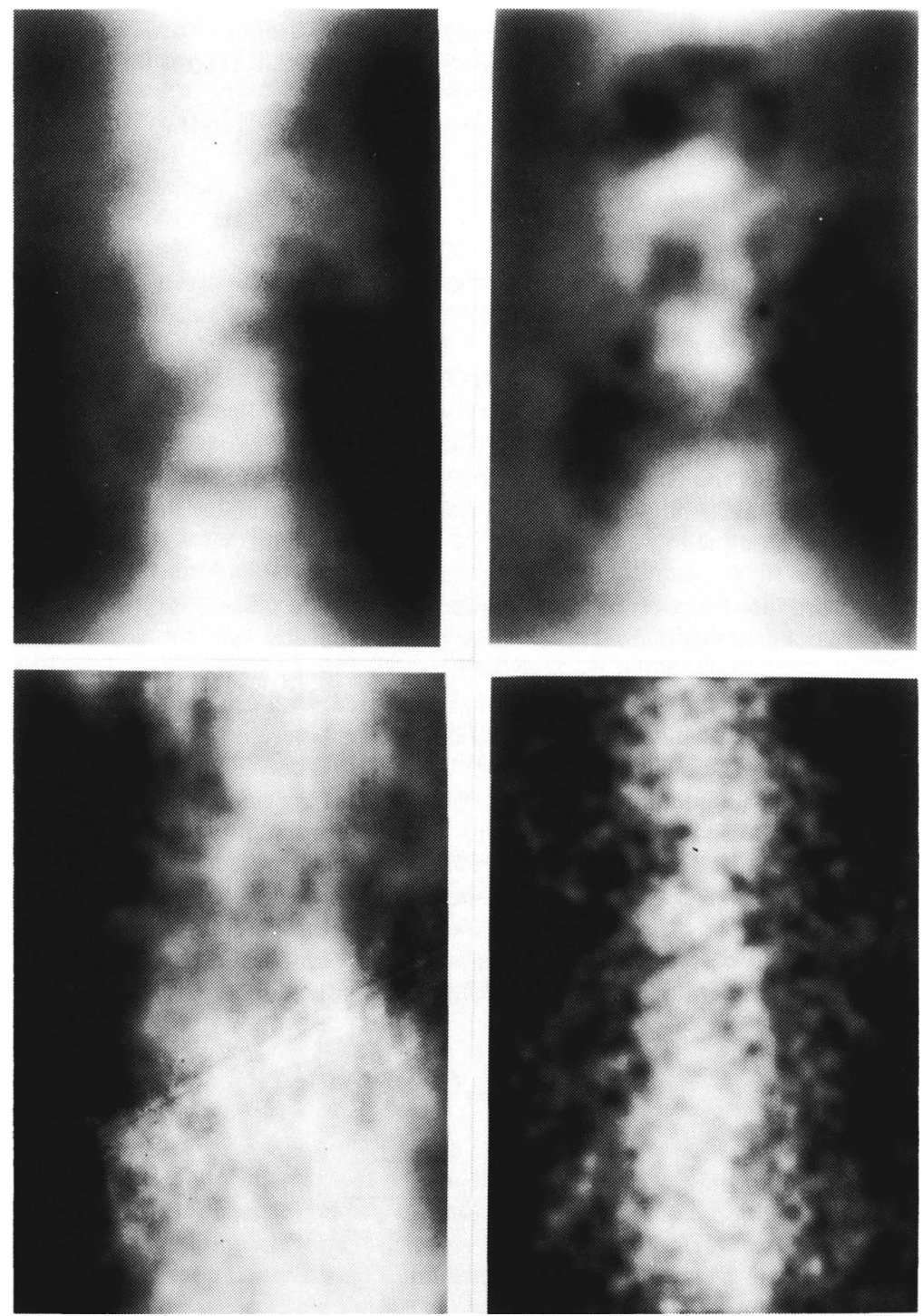


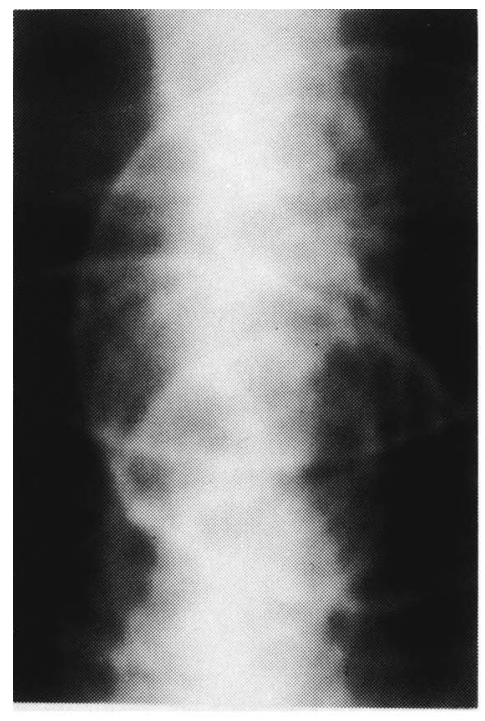

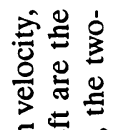

$\Xi \stackrel{0}{0}$

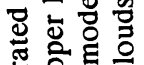
꼰 은응 总焉 仓气气气 灵光

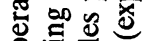
를을 옹

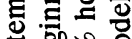

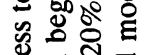

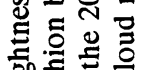

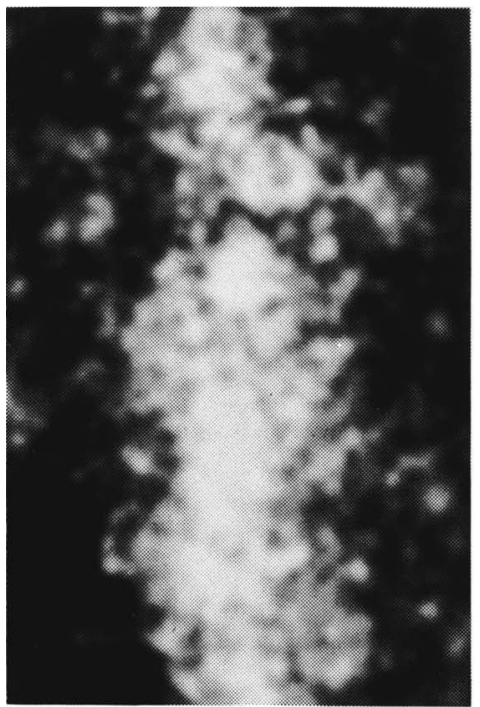
些要

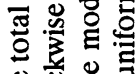
융 응

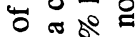
흘 吾号怘

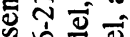

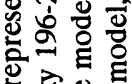
엃 응 웡 접유 토 范 政官

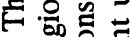
인 总

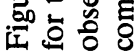



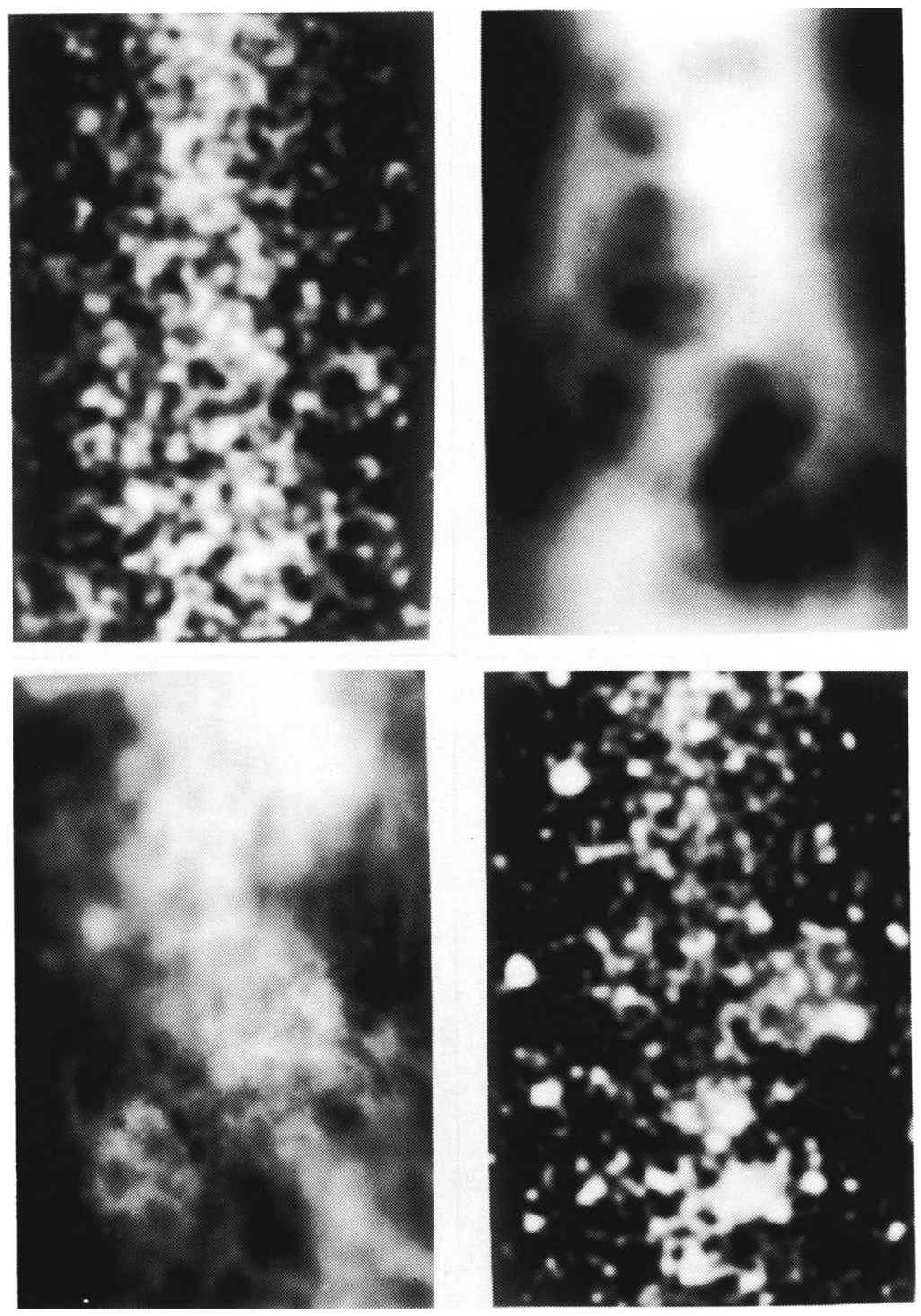


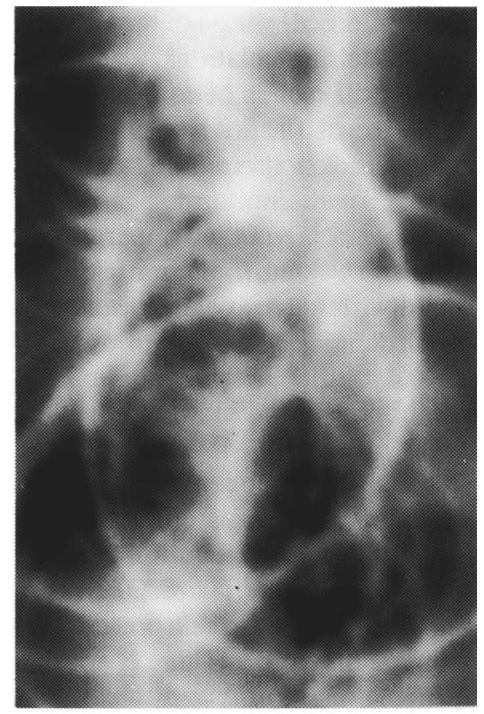

可 롫

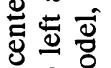

응으

דิ

웡

ฮ

范

등. 싱

$\Xi$ 토

o

웡

힌 을 몽

잉 잉

ఒँ

홍웡

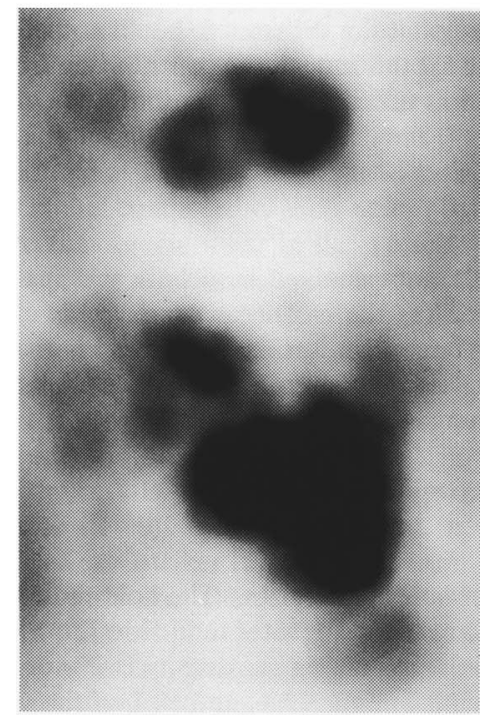

影证自 点的完

으웜 要专 늘 ธัँ

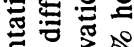
릉 议 흥.:응 웜 氙 突 첳ㅊ 해워 政 造. in 고트응 닉유 证光 


\subsection{Cloud Models}

Six different neutral hydrogen models were considered, although they can be classified either as cloud models or pervasive models. For the cloud models, calculations were made of (1) uniform spherical clouds of constant size, (2) nonuniform spherical clouds of constant size, and (3) two component clouds of varying sizes, which is representative of the McKee-Ostriker model. In all cases, the velocity dispersion of the clouds are taken to be $6 \mathrm{~km} \mathrm{~s}^{-1}$ and the same value is used for the internal velocity dispersion of the gas within a cloud. For the uniform spherical cloud model, the radius of the clouds is taken to be $5 \mathrm{pc}$, the density is $20 \mathrm{~cm}^{-3}$, and the temperature is $100 \mathrm{~K}$ unless otherwise stated. This is similar to the standard "Spitzer" cloud (Spitzer 1978). In the second model, the density decreases radially outward in a cloud with a density law given by

$$
n_{\text {cloud }}(r)=n_{\text {cloud }}(r=0) e^{-\frac{r}{r_{\text {cond }}}}
$$

where $\mathrm{r}_{\text {cloud }}=3 \mathrm{pc}$ and $\mathrm{n}_{\text {cloud }}(\mathrm{r}=0)=20 \mathrm{~cm}^{-3}$ (the exponential cloud model); the masses of these clouds and of the uniform clouds are the same.

The two-component cloud case, model 3 , has a cold uniform cloud $\left(\mathrm{T}=80 \mathrm{~K}, \mathrm{n}=42 \mathrm{~cm}^{-3}\right)$ surrounded by a larger warm low-density uniform cloud $\left(T=8000 \mathrm{~K}, \mathrm{n}=0.37 \mathrm{~cm}^{-3}\right)$. This model is suggested by McKee and Ostriker (1977), and following their prescription, the radius of the warm component is 2.5 times that of the cold component $\left(\mathrm{r}_{\text {cold }}\right)$. There is a range of cloud sizes where the number of clouds of a particular size is proportional to $\mathrm{r}^{-4}$, and the minimum and maximum values for $r_{\text {cold }}$ are $1.5 \mathrm{pc}$ and $10 \mathrm{pc}$ respectively. This minimum radius is a bit larger than the value suggested by McKee and Ostriker $(0.5 \mathrm{pc})$, but this difference should not lead to a qualitative change in the results. In calculating the opacity for this model, the contribution from the warm component is neglected, which is a good approximation since its optical depth is small along all lines of sight due to its high temperature.

\subsection{Continuous Models}

In contrast to the cloud models, we examined models in which there is a continuous $\mathrm{HI}$ distribution given by the above equation for the mean gas density plus either holes or holes and expanding shells in that distribution. The motivation for this model is that supernovae can create bubbles or superbubbles in which $\mathrm{HI}$ is converted into hot gas or displaced. Holes with a range of sizes exist in a single model, with the range of radii being given by

$$
0 p c<r_{\text {hole }}(R)<100 \frac{R}{R_{\odot}} p c
$$

On average, there are equal numbers of holes of all sizes. The hole radii are scaled to the galactic radius because the larger superbubbles are seen at greater radii (McCray and Kafatos 1987). When a hole is placed in the $\mathrm{HI}$ distribution, the remaining gas density is $2 \%$ of the original density.

Models were calculated in which $0 \%, 20 \%, 33 \%$, and $50 \%$ of the volume of the interstellar medium is occupied by holes. As the percentage of the volume in holes increases, so does the HI density in the remaining HI substrate (after all, we still reproduce the total observed emissivity).

The final model considered is where the material in the holes is placed in an expanding shell at the boundary of the shell. The expansion velocity is taken to be $30 \mathrm{~km} \mathrm{~s}^{-1}$ for all shells. Calculations were made when $20 \%$ and $33 \%$ of the volume of ISM is in the form of holes, which are surrounded by shells. 


\section{RESULTS}

There is a qualitative similarity between the cloud models, all of which provide a poor representation of the data (Fig. 1,2). This is especially evident in the channel maps of the cloud models in Figure 2 , where much of the flux is concentrated in a small fractional area. The striking difference between the observations and the cloud models that the power is dominant on small scales in the cloud models while in the observations, it occurs both on large and small scales; power spectrum analysis confirms this inference. This difference in the angular size distribution of the emission structure is greatest between the data and the two-component cloud models.

The reason for the apparent failure of the cloud models to reproduce the data is that there are too few clouds that contribute to each resolution element in a map (especially the channel maps). This problem is greatest in the low velocity channel maps (gas within 2-3 kpc of the Sun) and at latitudes greater than $2^{\circ}$. For this set of conditions, there are only a few clouds per resolution element (telescope beam), so the beam to beam variations can be large. This effect is greatest in the two-component cloud model, where the few large clouds can dominate the emission locally. Although it may be possible to reproduce some of the large-scale structure in the cloud models by introducing an agent that groups the clouds (e.g., supernovae), the problem with the small scale structure will remain. These calculations suggest that a theoretical understanding of the interstellar medium should not be based on cloud structures.

The continuous models of the ISM reproduce some of the characteristics of the data with greater success. In Figure 1, a comparison between the observations and the $20 \%$ and $30 \%$ hole models shows that the large-scale structure is naturally reproduced. The small-scale structure so dominant in the cloud models is much less pronounced in these continuous models (actually, there is too little power on small scales). Because the location of the holes are uncorrelated, they can occasionally line up over a velocity range comparable to the velocity dispersion of the gas $(0.5 \mathrm{kpc})$. When this occurs, there is a local minimum in the channel map (dark spot). Local minima of similar depth occur in the data and in the $20 \%$ hole model, but the variations seen in the $33 \%$ hole model are already becoming so large as to be inconsistent with the observations.

The continuous model composed of $20 \%$ holes plus shells shows very pronounced emission from shells, in contrast to the observations. In these calculations, the thickness of the shells is small so that when seen edge-on, they are always unresolved. At a typical distance of the shells in Figure 2 , any shell thinner than $15 \mathrm{pc}$ would be unresolved and appear like the shells in this calculation. Shells thinner than this are expected in both supernova and supershell calculations, although with less mass than we have employed. The infrequence of such features in the data indicate either that shells are much thicker than assumed, less frequent (not associated with every hole), or much less massive (most likely, a combination of all three effects).

To summarize the results of our models, the geometry of the interstellar medium is not described by an ensemble of clouds, either uniform, non-uniform (exponential), or the twocomponent clouds (the McKee-Ostriker ISM). The neutral ISM is better described by a continuous HI distribution with holes with sizes characteristic of both supernova remnants and superbubbles (diameters up to $200 \mathrm{pc}$ ). The amplitude of the large-scale variations $\left(>1^{\circ}\right)$ places limits on the volume occupied by these holes, suggesting that no more than about $30 \%$ of the ISM is in ionized material. This result is consistent with the observations of other ISM phases if about $10-20 \%$ of the volume is occupied by hot X-ray emitting gas and about $20 \%$ of the volume is occupied by the warm ionized component seen by Reynolds (some of this could be cospatial with the HI, so it might not require a displacement of the neutral phase). 


\section{ACKNOWLEDGEMENTS}

This project is deeply indebted to Jay Lockman for the use of his data and for his advice. JNB gratefully acknowledges support for this project from NASA LTSA-89-033.

\section{REFERENCES}

Brinks, E., and Bajaja, E. 1986, Astr. Ap., 169, 14

Brinks, E., and Shane, W.W. 1984, Astr. Ap. Suppl., 55, 179.

Cowie, L.L, McKee, C.F., and Ostriker, J.P. 1981, Ap. J., 247, 908.

Cox, D.P. 1981, Ap. J., 245, 534.

Cox, D.P., and Reynolds, R.J. 1987, Ann. Rev. Astr. Ap., 25, 303.

Cox, D.P., and Smith, B.W. 1974, Ap. J. (Letters), 189, L105.

Dickey, J.M., and Lockman, F.J. 1990, Annual Reviews of Astronomy and Astrophysics, 28, in press.

Deul, E.R. 1988, Ph.D. Thesis, Sterrewacht Leiden.

Heiles, C. 1984, Ap. J. Suppl., 55, 585.

Kulkarni, S.R., and Heiles, C. 1988, in "Galactic and Extragalactic Radio Astronomy", ed. G.L. Verschuur and K.I. Kellermann (Springer: Heidelberg), p. 95.

Lockman, F.J., and Ganzel, B.L. 1983, Ap. J., 268, 117.

Mac Low, M.-M., McCray, R. 1988, Ap. J., 324, 776.

Mac Low, M.-M., McCray, R., and Norman, M.L. 1989, Ap. J., 337, 141.

McCammon, D., and Sanders, W.T. 1984, Ap. J., 287, 167.

McCray, R., and Kafatos, M. 1987, Ap. J., 317, 190.

McKee, C.F., and Ostriker, J.P. 1977, Ap. J., 218, 148.

Norman, C.A., and Ikeuchi, S. 1989, Ap. J., 345, 372.

Reynolds, R.J. 1989, Ap. J. (Letters), 339, L29.

Spitzer, L. 1978, "Physical Processes in the Interstellar Medium", (Wiley: New York) p. 44.

Tenorio-Tagle, G., and Bodenheimer, P. 1988, Ann. Rev. Astr. Astrophys., 26, 145.

Tenorio-Tagle, G., Bodenheimer, P., and Rozyczka, M. 1987, Astron. Ap., 179, 219.

Tomisaka, K., and Ikeuchi, S. 1986, Pub. Astr. Soc. Japan, 38, 697.

Van Buren, D. 1986, Ap. J., 306, 538.

Weaver, R., Castor, J., McCray, R., Shapiro, P., and Moore, R. 1977, Ap. J., 218, 377. 